\title{
Therapeutic post-infection options for Ebola virus treatment set to broaden
}

\section{cef \\ all antibody- \\ treated \\ animals \\ survived}

The recent Ebola virus (EBOV) outbreak in West Africa highlighted the paucity of effective anti-viral therapies for this highly lethal disease. Numerous potential post-infection therapeutics are currently in development, and now a small molecule and a monoclonal antibody have each been shown to prevent manifestations of the disease in non-human primate models when administered up to 3 or 5 days after infection.

Writing in Nature, Warren and colleagues described GS-5734, a small-molecule prodrug of an adenosine analogue. In cells, the triphosphate metabolite of GS-5734 is thought to inhibit the production of viral proteins by acting as a substrate for the viral RNA-dependent RNA polymerase, and thus as an RNA-chain terminator. GS-5734 demonstrated broad antiviral activity in cell culture, inhibiting replication of various pathogenic filoviruses, including numerous strains of EBOV, Marburg and Bundibugyo virus, as well as other pathogenic RNA viruses, such as respiratory syncytial virus and Middle East respiratory syndrome virus.

In a lethal non-human primate model of acute EBOV infection, $100 \%$ of animals that received intravenous administration of GS-5734 (starting 3 days after virus exposure) survived, whereas untreated animals died within 9 days. The highest tested dose, $10 \mathrm{mg}$ per kg once daily, resulted in the strongest suppression of viral titer in plasma, and the virus became undetectable in the plasma of treated animals 12 days after viral challenge.

Meanwhile, Corti and colleagues isolated memory B cells from a survivor of the 1995 Ebola outbreak. Many of these clones produced monoclonal antibodies (mAbs) that bound the EBOV glycoprotein (GP), which is used for viral entry. Two clones with the highest potency (mAb100 and mAb114) prevented wild-type virus from infecting

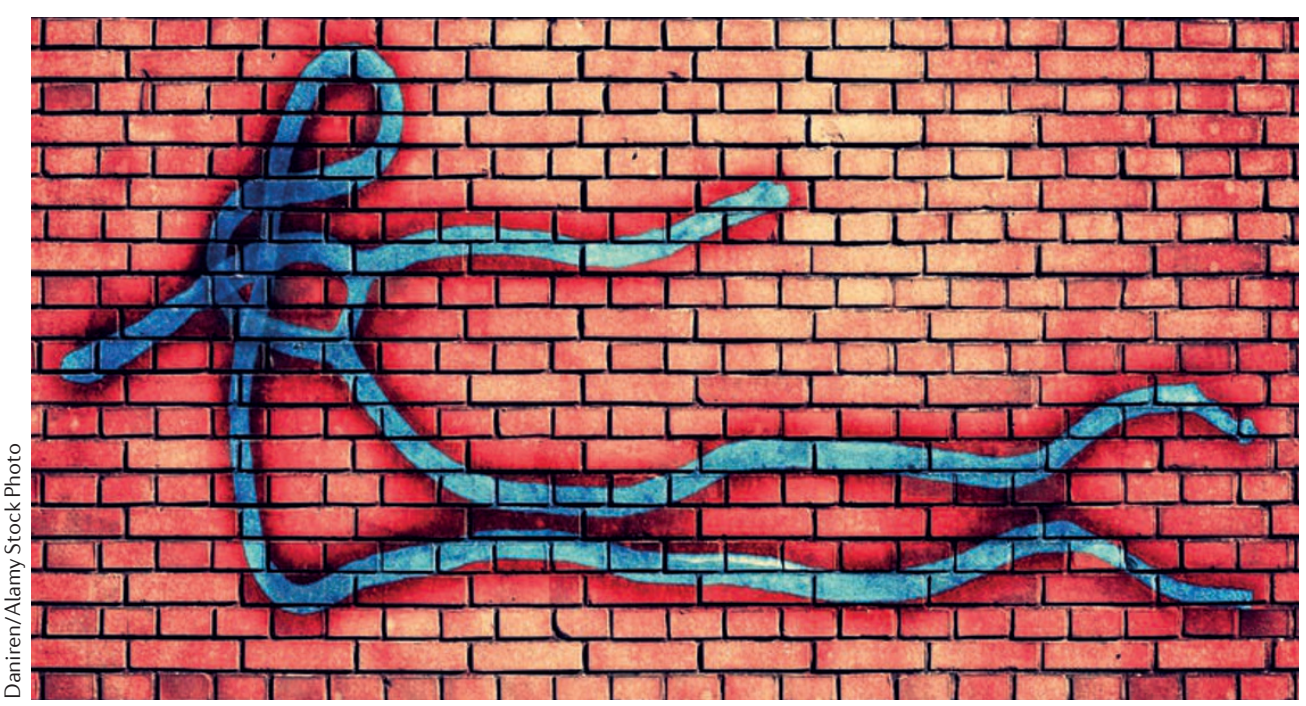

human HEK293T cells in culture and elicited antibody-dependent cell-mediated toxicity, a key viral clearance mechanism through which infected cells are identified and lysed. Importantly, mAb100 and mAb114 were found to bind to different regions of GP with novel mechanisms of neutralization; mAb100 prevented GP maturation and $\mathrm{mAb} 114$ prevented the virus from binding its receptor.

To investigate these antibodies in non-human primates, animals received daily injections of either the two-mAb combination starting 1 day after viral challenge or mAb114 alone 1 day or as late as 5 days after virus exposure. The vehicle-treated animal died within 10 days; all antibody-treated animals survived. Those treated with both antibodies had no detectible viraemia, whereas virus was transiently detected in the blood of animals treated with mAb114 monotherapy, but these animals remained free of Ebola disease symptoms.

Although the most recent and largest Ebola outbreak has come to an end, there are still small clusters of new infections emerging in West Africa, and these two agents could form the foundation of new therapies that would be crucial if and when the virus re-emerges.

Megan Cully

ORIGINAL ARTICLES Warren, T. K. et al. Therapeutic efficacy of the small molecule GS-5734 against Ebola virus in rhesus monkeys. Nature 531, 381-385 (2016) | Corti, D. et al. Protective monotherapy against lethal Ebola virus infection by a potently neutralizing antibody. Science 351, 1339-1342 (2016) FURTHER READING Misasi, J. et al. Structural and molecular basis for Ebola virus neutralization by protective human antibodies. Science $\mathbf{3 5 1}$, 1343-1346 (2016) 